\title{
Effects of passive leg raising and volume expansion on mean systemic pressure and venous return in shock in humans
}

\author{
L Guérin*, JL Teboul, R Persichini, M Dres, C Richard, X Monnet \\ From ESICM LIVES 2015 \\ Berlin, Germany. 3-7 October 2015
}

\begin{abstract}
Introduction
The passive leg raising (PLR) test has been developed for predicting fluid responsiveness. Nevertheless, one could question its ability to induce a significant increase in the stressed blood volume. Also, the fact that some patients do not respond to volume expansion by augmenting cardiac output could theoretically be related to the absence of preload dependence, but also to an insufficient increase in stressed blood volume.
\end{abstract}

\section{Objectives}

To assess the effects of increases in preload induced by passive leg raising (PLR) and fluid infusion on the pressure gradient between mean systemic pressure (Psm) and central venous pressure (CVP) and on the resistance to venous return (Rvr).

\section{Methods}

In 30 patients with an acute circulatory failure, in order to estimate the venous return curve, we constructed the regression line between pairs of cardiac index (CI) and CVP. Values were measured during end-inspiratory and end-expiratory ventilatory occlusions performed at two levels of positive end-expiratory pressure. The $\mathrm{x}$-axis intercept was used to estimate Psm and the inverse of the slope to quantify Rvr. These measurements were obtained at baseline, during PLR and after fluid infusion.

\section{Results}

In volume-responders $(\mathrm{n}=15)$, Psm increased from $25 \pm$ 13 to $31 \pm 13 \mathrm{mmHg}$ during PLR and to $32 \pm 17 \mathrm{mmHg}$ after fluid infusion. CVP increased from $7 \pm 3 \mathrm{mmHg}$ to
$9 \pm 4 \mathrm{mmHg}$ during PLR and to $9 \pm 4 \mathrm{mmHg}$ after fluid infusion. Thus, in these patients, the Psm-CVP gradient increased significantly during PLR and fluid infusion. By contrast in non-volume responders, PLR and fluid infusion increased Psm and CVP to a similar extent, so that the Psm-CVP gradient was unchanged. Rvr was modified neither by PLR nor by fluid infusion in both groups.

\section{Conclusions}

In preload-dependent patients, PLR and fluid infusion increased venous return owing to an increase in Psm higher than in CVP, with unchanged Rvr. In preloadindependent patients, neither PLR nor fluid infusion changed venous return, Psm-CVP gradient and Rvr.

Published: 1 October 2015

\section{doi:10.1186/2197-425X-3-S1-A16}

Cite this article as: Guérin et al:: Effects of passive leg raising and volume expansion on mean systemic pressure and venous return in shock in humans. Intensive Care Medicine Experimental 2015 3(Suppl 1):A16. 